\title{
Radiation therapy as part of the therapeutic regimen for extensive multilocular myxedema in a patient with exophthalmos, myxedema and osteoarthropathy syndrome: A case report
}

\author{
KHALED ELSAYAD ${ }^{1}$, JAN KRIZ $^{1}$, JULIA BAUCH ${ }^{1}$, SERGIU SCOBIOALA ${ }^{1}$, \\ UWE HAVERKAMP $^{1}$, CORD SUNDERKÖTTER ${ }^{2}$ and HANS THEODOR EICH ${ }^{1}$ \\ Departments of ${ }^{1}$ Radiation Oncology and ${ }^{2}$ Dermatology, University Hospital of Muenster, Muenster D-48149, Germany
}

Received May 20, 2014; Accepted February 13, 2015

DOI: $10.3892 / \mathrm{ol} .2015 .2990$

\begin{abstract}
Exophthalmos, myxedema and osteoarthropathy (EMO) comprise the triad known as EMO syndrome, which is rarely observed in patients with autoimmune thyroid disease. The present study reports the case of a patient with EMO, including the response of this rare combination to radiotherapy. A 48-year-old patient with EMO syndrome presented to the Department of Radiation Oncology, University Hospital of Muenster, eight years prior to writing with therapy-resistant pretibial myxedema and hypertrophic osteoarthropathy of the metacarpal bones. The patient had been diagnosed with Graves' disease (GD) 26 years prior to presentation, which was treated by thyroidectomy and radioiodine therapy. Four years subsequent to the diagnosis of GD, the patient developed exophthalmos, which was treated using radiotherapy. An evident pretibial, foot and hand myxedema developed within the 10 years following the onset of orbitopathy. The skin lesions were treated using radiation therapy subsequent to the failure of multiple surgical procedures and medical treatments. Almost eight years subsequent to the administration of irradiation, no recurrence was observed on the lower right leg, nor was any recurrence on the lower left leg observed approximately four years subsequent to the completion of radiotherapy. However, an additional lesion on the left hand demonstrated slow progression following treatment with radiation therapy. The present study indicates that radiation therapy can be considered as adjuvant therapy for patients with refractory myxedema, to prevent or delay the recurrence of myxedema subsequent to surgical excision.
\end{abstract}

Correspondence to: Mr. Khaled Elsayad, Department of Radiation Oncology, University Hospital of Muenster, Building A1, 1 Albert-Schweitzer-Campus, Muenster D-48149, Germany

E-mail: khaled.elsayad@uni-muenster.de

Key words: EMO syndrome, exophthalmos, myxedema, osteoarthropathy, Graves' disease, radiotherapy

\section{Introduction}

Myxedema is a thyroid dermopathy that occurs as a component of exophthalmos, myxedema and osteoarthropathy (EMO) syndrome or as an extrathyroidal complication of Graves' disease (GD). The dermopathy is characterized by a localized skin thickening that is confined in $~ 99 \%$ of cases to the area of the shins and, in rare cases, to the upper limbs and eyelids (1). Myxedema is an uncommon manifestation of autoimmune thyroid disease and occurs more often in patients with hyperthyroidism, with $91 \%$ of cases arising in patients with hyperthyroidism. Myxedema may develop in hypothyroidism subsequent to therapy for GD (1), and appears extremely rarely in Hashimoto's thyroiditis without thyrotoxicosis, and even in euthyroid patients (1-3). In GD, myxedema, together with ophthalmopathy, is one of the extrathyroidal manifestations of the disease, in addition to hyperthyroidism secondary to diffuse goiter (4). When myxedema occurs as part of a triad of extrathyroidal manifestations that also includes exophthalmos and osteoarthropathy, it constitutes EMO syndrome, which is observed in $<1 \%$ of patients with autoimmune thyroid disease (5). The extrathyroidal symptoms of EMO tend to develop chronologically with an onset of eye disease followed by myxedema and then finally osteoarthropathy or acropachy in cases of a long duration (6-8).

Clinically, myxedema presents with non-pitting edema, nodules or plaques. In severe cases, the dermopathy may progress to elephantiasis. The mean age at diagnosis of pretibial myxedema is 53 years, with a female to male ratio of $4: 1$ (1).

Pathophysiologically, myxedema is characterized by the accumulation of glycosaminoglycans in the dermis and panniculus adiposus that compresses the dermal lymphatics, resulting in dermal edema and the clinical features of lymphedema $(2,9)$.

The thyrotropin receptor (TSH-R) has been nominated as a common target antigen in Graves' ophthalmopathy (GO) and pretibial myxedema (PTM) (10-12). In previous studies, the presence of TSH-R immunoreactivity has been also reported to be involved in the muscles and adipose tissues of patients with GO and in the dermis of patients with PTM $(6,10)$. Therefore, the antigen-antibody reaction is hypothesized 
to result in the stimulation of fibroblasts, with a secondary release of cytokines leading to lymphocyte attraction and production of glycosaminoglycans, eventually causing tissue swelling $(2,10,11)$.

The cutaneous manifestations of hyperthyroidism include warm, moist and pruritic skin. Ophthalmopathy in GD is characterized by the swelling of rectus muscles, connective tissue expansion in the posterior orbit that results in the protrusion of the eyes, extraocular muscle dysfunction and impaired vision in advanced cases, due to compressive neuropathy of the optic nerve $(13,14)$. Thyroid acropachy is a rare clinical presentation of autoimmune thyroid disease and manifests with clubbing and swelling of the digits due to osteoarthropathy and periosteal reaction of the bones of the upper or lower extremities $(1,11)$.

The majority of patients with mild myxedema do not require treatment, with $50 \%$ of cases concluding in spontaneous remission. Moderate myxedema may require treatment in patients that are symptomatic and experience pain, impaired motion of the joints or disfigurement (2). The present study reports the case of a patient with EMO syndrome that suffered from extensive myxedema and was treated using radiotherapy as a component of the therapeutic regimen. Written informed consent was obtained from the patient.

\section{Case report}

A 48-year-old male patient was diagnosed with thyrotoxicosis secondary to GD at his local clinic (Lengerich, Germany). A complete check-up revealed elevated levels of thyroid stimulating hormone receptor antibody (TSHR-Ab). The patient was initially treated with a subtotal thyroidectomy in April 1987, and then with radioiodine therapy in 1989 at the Department of Nuclear Medicine, University Hospital of Muenster (Muenster, Germany). The patient also demonstrated mild bilateral pretibial myxedema, which disappeared within 17 months following radioiodine therapy. Subsequently, the patient developed hypothyroidism, which was compensated for through the daily administration of $250 \mu \mathrm{g}$ L-thyroxine. The dose of L-thyroxine was then gradually reduced over the period of one year to $175 \mu \mathrm{g}$.

Whilst the patient was receiving $175 \mu \mathrm{g}$ L-thyroxine daily, an euthyreotic condition was identified, with the following thyroidal values being obtained: TSH $1.23 \mu$ units $/ \mathrm{ml}$ (normal range, 0.35-4.50 $\mu$ units $/ \mathrm{ml}$ ); free triiodothyronine, $3.10 \mathrm{pg} / \mathrm{ml}$ (normal range, 2.0-4.50 pg/ml); free thyroxine, $1.33 \mathrm{ng} / \mathrm{dl}$ (normal range, 0.70-1.65 ng/ml); thyroglobulin $\mathrm{Ab}, 10.1$ units/ml (normal level, <1.0 units/ml); TSHR-Ab, 69.6 units/1 (normal level, $<3.0$ units/l); and thyroid peroxidase antibody, 14.4 units/ml (normal level, $<2.0$ units $/ \mathrm{ml}$ ).

In 1993, the patient developed exophthalmos, which was successfully treated by the administration of conventional radiotherapy at a total dose of $36 \mathrm{~Gy}$. Laser-based patient positioning was used to ensure precise positioning prior to each radiation session (15). The patient experienced mild transient conjunctivitis and periorbital erythema subsequent to the treatment.

In 2000, a myxedema began to develop on the right hand, and then progressed to involve the lower right leg. Two
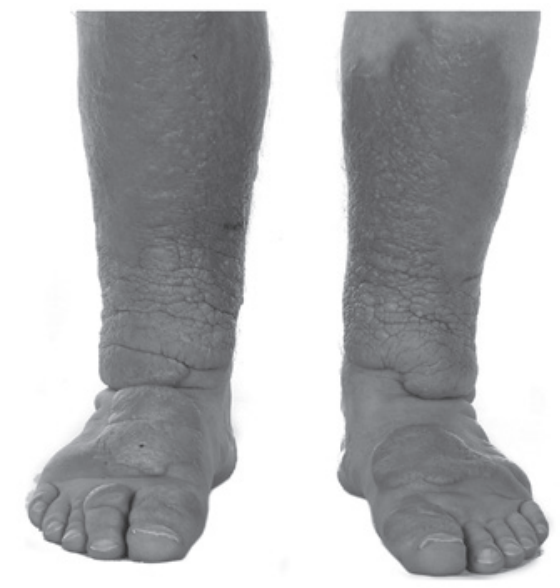

Figure 1. Anterior view of the legs prior to treatment, revealing extensive thickened plaques extending around the legs and clubbing of the toes.

years later, the left hand and then the lower left leg were also affected. The patient was treated using a variety of medications, consisting of the administration of topical and systemic corticosteroids, cyclophosphamide, hydroxychloroquin and everolimus, as a trial to improve his skin condition by decreasing the fibroblast production of hyaluronic acid. Compression bandaging and regular lymphatic drainage were also administered. Subsequent to these therapies, no sufficient improvement had been observed and the patient was therefore referred to the Department of Radiation Oncology at the University Hospital of Muenster in 2005.

The clinical examination performed prior to radiotherapy confirmed the presence of massive pretibial myxedema in the form of non-pitting edema and skin plaques, combined with areas of extensive fibrosis (Fig. 1). Nodular tumors on the dorsal aspect of the hands and feet were clinically apparent and resulted in a severe cosmetic impairment, as well as discomfort due to continuous pressure from footwear and impaired motion of the ankles. At the time of the diagnosis of myxedema, the patient was non-thyrotoxic. The histological examination of the myxedematous pretibial and hand lesions revealed a typical deposition of mucin accompanied by fibrosis of collagenous fibers and interstitial mucopolysaccharides. Perivascular infiltration of lymphocytes was also noted. The epidermis was found to be irregularly acanthotic with compact hyperkeratosis.

As the patient had developed thyroid acropathy over the course of the disease, with clubbing of the fingers and toes being demonstrated, and had experienced a periosteal reaction in the metacarpal bones and osteoarthropathy, as revealed by an X-ray of the hands performed in 2002, the triad of exophthalmos, myxedema and osteoarthropathy was fulfilled and a diagnosis of EMO syndrome was established. Table I reports the details of the radiation doses administered to the patient and the clinical outcomes.

In February 2005, radiation therapy was commenced to treat the lower left leg and foot at a total dose of 5 Gy. Simultaneously, radiotherapy of the lower right leg and foot was commenced, with the administration of $\leq 20 \mathrm{~Gy}$, due to severe skin myxedema. Three months later, an additional 16 Gy was administered to the lower right leg due to the persistence of 


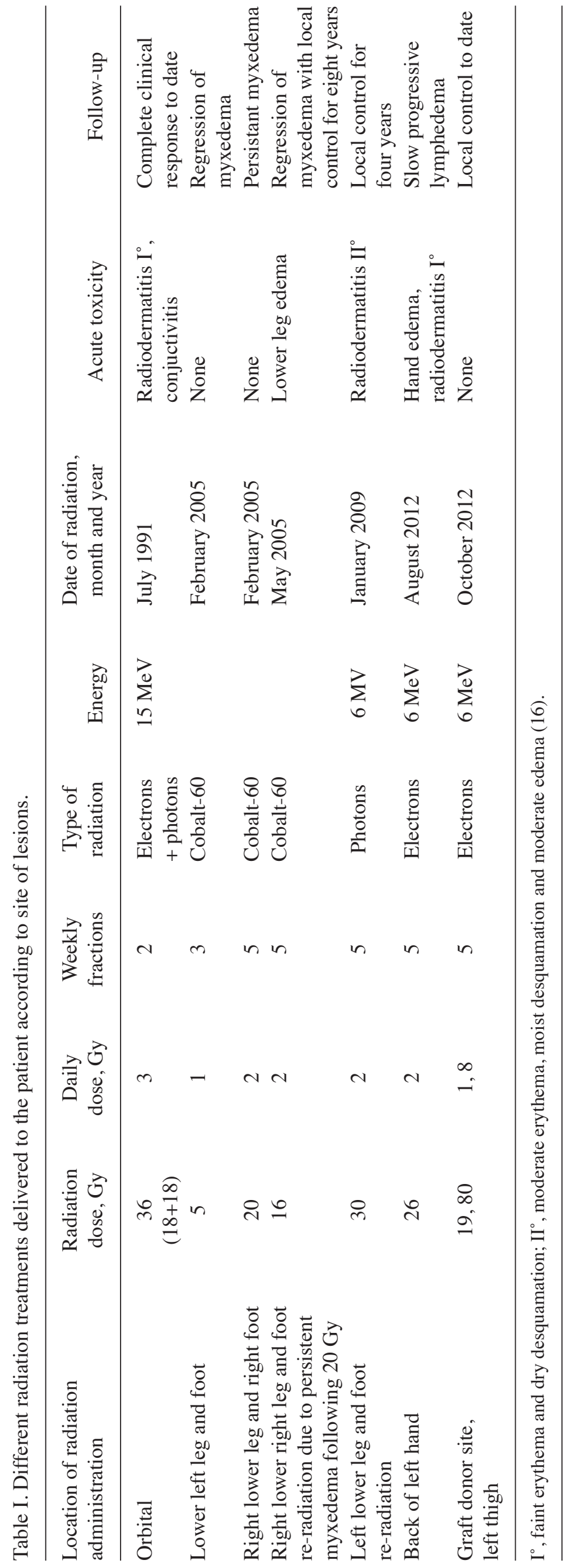




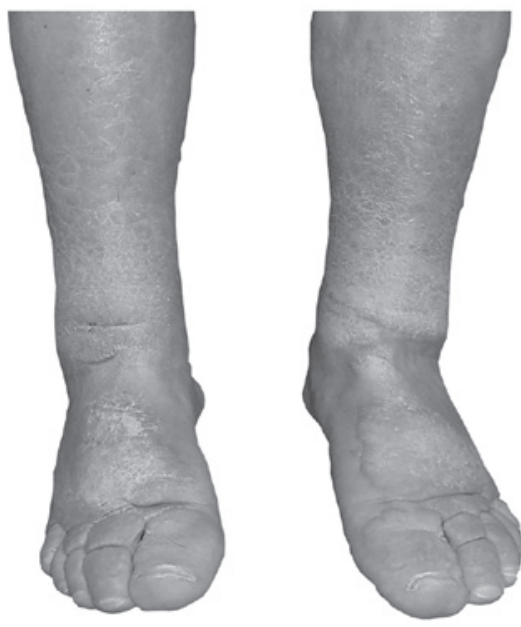

Figure 2. Anterior appearance of the legs subsequent to treatment, demonstrating the decreased edema, fibrosis and nodularity.

myxedema. The radiotherapy was well tolerated, with the exception of skin edema and slight erythema occurring on the right side a few days subsequent to the completion of radiotherapy. However, this resolved spontaneously after three weeks.

In November 2008, due to skin disfigurement, the patient was treated with surgical shave excision on the lower legs, which was performed by dermatological surgeons. Subsequent to the surgery on the left leg, adjuvant radiotherapy was commenced up to a total dose of $30 \mathrm{~Gy}$ (Fig. 2). Since 2009, the present patient has received $400 \mathrm{mg}$ pentoxifylline daily as maintenance therapy to reduce the extent of fibrotic lesions induced by radiation therapy (17).

In February 2010, due to the presence of severe thyroid myxedema on the back of the left hand, the skin was resected and the patient developed myxedematous recurrence after seven months. In June 2012, the Departments of Dermatology and Radiation Oncology jointly decided to perform wide skin excision and skin transplantation on the back of the left hand and then to perform radiation treatment at a dose of 26 Gy for consolidation. Slight skin edema and erythema were noted. Five weeks later, the graft donor site exhibited myxedematous skin changes, four months subsequent to skin transplantation, in the form of skin elevation and swelling. These symptoms were also treated with radiotherapy at a dose of $19.8 \mathrm{~Gy}$.

Subsequent to the completion of radiotherapy, the skin erythema had clearly improved. The patient was highly satisfied with the results of treatment. Nearly eight years subsequent to irradiation, no recurrence of myxedema was observed on the lower right leg. On the lower left leg no recurrence was observed approximately four years subsequent to the completion of radiotherapy. At the final follow-up examination six months prior to writing, slight lower leg swelling was observed on each side, which was considered to be recurrent myxedema. At present, this recurrence continues to be treated locally with compression bandaging. Additional lesions on the left hand and thigh due to skin transplantation were also stable subsequent to the completion of radiation treatments. The left hand demonstrates the presence of slowly progressing lymphedema, nearly six months subsequent to radiotherapy, which is also currently being treated with compression bandaging.

\section{Discussion}

The mechanism of myxedema is not completely clear, and it is assumed that the presence of the TSHR-Ab stimulates fibroblasts, leading to glycosaminoglycans production and resulting in the engorgement of the involved tissues $(10,11)$. Mild cases of myxedema or cases without cosmetic or local functional limitation do not require topical treatment and are usually followed up by endocrinologists. The administration of topical corticosteroids coupled with occlusive or compressive dressings may be used to treat symptomatic cases (2). The treatment of refractory cases is usually suppressive in order to control symptoms without curative intent. Other management options have been advocated. Shinohara et al described success using intra-lesional octreotide for $>15$ months (18). Felton et al successfully treated a patient using a combination of surgical and octreotide treatment, resulting in local control of skin lesions lasting more than nine years (19). Jolles et al reported the clinical improvement of pretibial myxedema in patients treated using high dose intravenous immunoglobulin therapy (20).

To the best of our knowledge, the present study reports the first case of extensive multilocular myxedema in a patient with EMO syndrome that was treated using radiotherapy. The skin lesions resulting from EMO syndrome in the present patient resulted in severe cosmetic and local functional impairment. The adjuvant radiotherapy administered subsequent to skin excision produced a significantly improved long-term local control of the skin lesions and improved ankle movements on each side. In addition to the radiation sessions, psychosocial support for the present patient aided in the development of coping strategies for the medical condition (21).

In the present patient, no exophthalmos was observed for 20 years subsequent to the administration of orbital conventional radiotherapy using two opposing lateral fields. In addition, radiotherapy has been used successfully for $>50$ years for the treatment of GO. At present, orbital radiation is performed using intensity-modulated radiotherapy to decrease incidence of complications. Low dose orbital radiotherapy of 16-20 Gy over two to three weeks was revealed to be effective in the treatment of GO $(22,23)$.

In summary, the routine use of radiation therapy for EMO syndrome is not recommended, in particular for the treatment of young patients with minimal cutaneous changes. It was also hypothesized that radiotherapy, in combination with surgical intervention, should be alternative modalities for those patients who exhibit recurrent or refractory myxedematous lesions with severe cosmetic disfigurement.

The present study reports the case of a patient that experienced EMO syndrome in addition to extensive myxedema, which demonstrated a good long-term clinical response to radiotherapy in combination with surgical procedures. The current study reveals that radiation therapy may be considered as an adjuvant therapy for patients with refractory myxedema in order to prevent or delay the recurrence of myxedema subsequent to surgical excision. Management 
of EMO syndrome requires discussion within a multidisciplinary team to determine individualized treatment options for each clinical presentation of the syndrome.

\section{Acknowledgements}

The authors would like to thank Susan Nieschlag for the language editing performed on the original manuscript.

\section{References}

1. Schwartz KM, Fatourechi V, Ahmed DD and Pond GR Dermopathy of Graves' disease (pretibial myxedema): Long-term outcome. J Clin Endocrinol Metab 87: 438-446, 2002.

2. Fatourechi V: Pretibial myxedema: Pathophysiology and treatment options. Am J Clin Dermatol 6: 295-309, 2005.

3. Chen JJ and Ladenson PW: Euthyroid pretibial myxedema. Am J Med 82: 318-320, 1987.

4. Brent GA: Clinical practice. Graves' disease. N Engl J Med 358: 2594-2605, 2008.

5. Anderson CK and Miller OF III: Triad of exophthalmos, pretibial myxedema, and acropachy in a patient with Graves' disease. J Am Acad Dermatol 48: 970-972, 2003.

6. Bahn RS, Dutton CM, Heufelder AE and Sarkar G: A genomic point mutation in the extracellular domain of the thyrotropin receptor in patients with Graves' ophthalmopathy. J Clin Endocrinol Metab 78: 256-260, 1994.

7. Saito S, Sakurada T, Yamamoto M, Yamaguchi T, Yoshida K, Sasai Y and Yoshinaga K: Exophthalmus-myxoedema circumscriptum praetibiale-osteoarthropathia hypertrophicans (E.M.O.) syndrome in Graves' disease: A review of eight cases reported in Japan. Tohoku J Exp Med 115: 155-165, 1975.

8. Senel E and Güleç AT: Euthyroid pretibial myxedema and EMO syndrome. Acta Dermatovenerol Alp Pannonica Adriat 18: 21-23, 2009.

9. Rongioletti F, Donati P, Amantea A, Ferrara G, Montinari M, Santoro F and Parodi A: Obesity-associated lymphoedematous mucinosis. J Cutan Pathol 36: 1089-1094, 2009.

10. Daumerie C, Ludgate M, Costagliola S and Many MC: Evidence for thyrotropin receptor immunoreactivity in pretibial connective tissue from patients with thyroid-associated dermopathy. Eur J Endocrinol 146: 35-38, 2002.

11. Fatourechi V, Ahmed DD and Schwartz KM: Thyroid acropachy: Report of 40 patients treated at a single institution in a 26 -year period. J Clin Endocrinol Metab 87: 5435-5441, 2002.
12. Stadlmayr W, Spitzweg C, Bichlmair AM and Heufelder AE: $\mathrm{TSH}$ receptor transcripts and TSH receptor-like immunoreactivity in orbital and pretibial fibroblasts of patients with Graves' ophthalmopathy and pretibial myxedema. Thyroid 7: 3-12, 1997.

13. Prabhakar BS, Bahn RS and Smith TJ: Current perspective on the pathogenesis of Graves' disease and ophthalmopathy. Endocr Rev 24: 802-835, 2003.

14. Bahn RS: Clinical review 157: Pathophysiology of Graves' ophthalmopathy: the cycle of disease. J Clin Endocrinol Metab 88: 1939-1946, 2003.

15. Stieler F, Wenz F, Scherrer D, Bernhardt M and Lohr F: Clinical evaluation of a commercial surface-imaging system for patient positioning in radiotherapy. Strahlenther Onkol 188: $1080-1084,2012$

16. Common Terminology Criteria for Adverse Events (CTCAE) Version 4.0. http://evs.nci.nih.gov/ftp1/CTCAE/ CTCAE_4.03_2010-06-14_QuickReference_8.5x11.pdf. Accessed August 30, 2013.

17. Delanian S, Porcher R, Rudant J and Lefaix JL: Kinetics of response to long-term treatment combining pentoxifylline and tocopherol in patients with superficial radiation-induced fibrosis. J Clin Oncol 23: 8570-8579, 2005.

18. Shinohara M, Hamasaki Y and Katayama I: Refractory pretibial myxoedema with response to intralesional insulin-like growth factor 1 antagonist (octreotide): Downregulation of hyaluronic acid production by the lesional fibroblasts. Br J Dermatol 143: 1083-1086, 2000

19. Felton J, Derrick EK and Price ML: Successful combined surgical and octreotide treatment of severe pretibial myxoedema reviewed after 9 years. Br J Dermatol 148: 825-826, 2003.

20. Jolles $S$ and Hughes J: Use of IGIV in the treatment of atopic dermatitis, urticaria, scleromyxedema, pyoderma gangrenosum, psoriasis, and pretibial myxedema. Int Immunopharmacol 6: 579-591, 2006

21. Schiel RO, Herzog W, Hof H, Debus J, Friederich HC, Brechtel A, Rummel J, Freytag P and Hartmann M: [Effect of systematic information about psychosocial support services during outpatient radiotherapy. A controlled trial]. Strahlenther Onkol 189: 579-585, 2013 (In German).

22. Nguyen NP, Krafft SP, Vos P, et al: Feasibility of tomotherapy for Graves' ophthalmopathy: Dosimetry comparison with conventional radiotherapy. Strahlenther Onkol 187: 568-574, 2011.

23. Staar S, Eich $H$ and Seegenschmiedt M: Graves' Orbitopathy. In: Radiotherapy for Non-Malignant Disorders. Seegenschmiedt M and Makoski H-B (eds). Brady L, Springer Berlin Heidelberg, Trott, K-R, pp469-486, 2008. 